\title{
Patterns close to the critical field in type-I superconductors
}

\author{
Mariela Menghini* and Rinke J. Wijngaarden \\ Department of Physics and Astronomy, Faculty of Sciences, Vrije Universiteit, De Boelelaan 1081, 1081HV Amsterdam, The Netherlands
}

(Received 31 July 2006; revised manuscript received 19 November 2006; published 31 January 2007)

\begin{abstract}
We study the patterns formed in the intermediate state of type-I superconducting lead $(\mathrm{Pb})$ slabs close to the transition to the normal state. Magneto-optical images reveal avalanchelike propagation of superconducting stripes in a normal matrix when decreasing the field while they transform into superconducting bubbles when increasing the field. Our results show that the combination of superconducting stripe width and its field dependence is not compatible with any of the present theoretical models. The stability of the structures is studied by modulating the magnetic field periodically in time. We find that close to the transition to the normal state a structure composed of superconducting bubbles is closer to equilibrium than a stripe pattern. We also observe that when the field is subsequently decreased the bubble pattern, as in other systems, transforms into a stripe one by continuous elongation and bending and only rarely by branching.
\end{abstract}

DOI: 10.1103/PhysRevB.75.014529

PACS number(s): 74.25.Ha, 89.75.Kd

\section{INTRODUCTION}

Type-I superconductors belong to the wide variety of systems where competing interactions lead to the formation of spatially modulated structures. Some other systems that share this property are ferrofluids, ferrimagnetic garnets and monomolecular amphiphilic films. ${ }^{1}$ The most common types of morphology that are encountered are stripe and bubble patterns. The stripes can be parallel and arranged in a periodic way or in a more disordered labyrinthine structure while, in ideal systems, the bubbles will form an hexagonal structure. The competition between a positive surface energy and the repulsive long-range dipolar interaction determines the equilibrium configuration for different external conditions. However, in this type of system there are several configurations of similar free energy and in general the system gets locked in one of these metastable states and truly equilibrium is never reached. This may be aggravated by even a small amount of spatially inhomogeneous quenched disorder. For certain systems, the region of stability of one or other type of structure has been calculated., ${ }^{2,3}$ Experimentally, the transition between stripe and bubble phases has been observed in many cases. ${ }^{1,4-6}$

In type-I superconductors the modulated phase consists of alternating normal $(\mathrm{N})$ and superconducting $(\mathrm{SC})$ regions and appears below a temperature dependent critical magnetic field, $H_{c}(T)$. This phase is known as the intermediate state (IS). Above $H_{c}(T)$ the sample is in the normal state (NS) and consequently in an homogeneous phase. The first theoretical treatment of the modulated phase was made by Landau ${ }^{7}$ who assumed that a slab sample in the IS will break in alternating straight SC and N parallel lamina. Within this model, the period of the domain structure can be calculated as a function of applied field. Experimentally, the Landau domain structure is obtained when the magnetic field is applied at small angles with respect to the large surface of a slab sample (Sharvin geometry). ${ }^{8}$ On the other hand, when the field is applied perpendicular to the surface of the sample the distribution of flux inside the sample forms rather more complicated structures. ${ }^{9}$ Very good agreement of the observed periodicity of the structure with the prediction of the Landau model has been found provided that the SC and N laminas are reasonable parallel..$^{9,10}$ Later on, Goren and Tinkham proposed $^{11}$ a theoretical model where the IS consists of an hexagonal array of normal bubbles. From their calculation it turned out that the bubble pattern has approximately the same energy as the laminar structure. This result indicates that slight variations in the sample characteristics as well as in the path followed in the $H-T$ phase diagram can be decisive in the pattern selection process. By means of Hall probe measurements in samples with low pinning, the authors found ${ }^{11}$ corroboration of the temperature, field and thickness dependence of the density of flux bubbles predicted by their theory.

In order to make a more accurate description of the formation of domains of arbitrary geometry, a current loop (CL) model that considers the IS as a collection of current ribbons flowing along the SC-N interfaces, was presented in Ref. 12. In this model the self-interaction of currents in a single domain as well as the interaction, as in free space, between different domains is considered. The current loop model is able to reproduce the results of the Landau model for the equilibrium period of straight laminae. Besides that, the instabilities of a circular flux domain (a flux bubble) and of a flux stripe are studied within this model. Very recently, ${ }^{13}$ an extension of the current loop model has been proposed in order to determine whether the flux stripe or bubble pattern correspond to the equilibrium configuration as a function of applied field. This constrained current loop (CCL) model includes the screening currents flowing in the top and bottom surface of the sample. Within this model, the characteristic length scales (stripe width and bubble diameter) can be calculated. For $H<0.8 H_{c}$ the experimental values of flux stripes width as a function of field are well described by the model. On the other hand, the bubble diameter is only fairly described in the limit $H \rightarrow 0$ for a variety of samples. Finally, a transition from the flux bubble phase to the stripe flux pattern as the field is increased is predicted consistently with the experimental observations, as we will now discuss in more detail.

It has been observed ${ }^{6,11,14-16}$ that an assembly of normal bubbles that span the thickness of the sample is formed upon flux penetration at low fields. The formation of the flux 

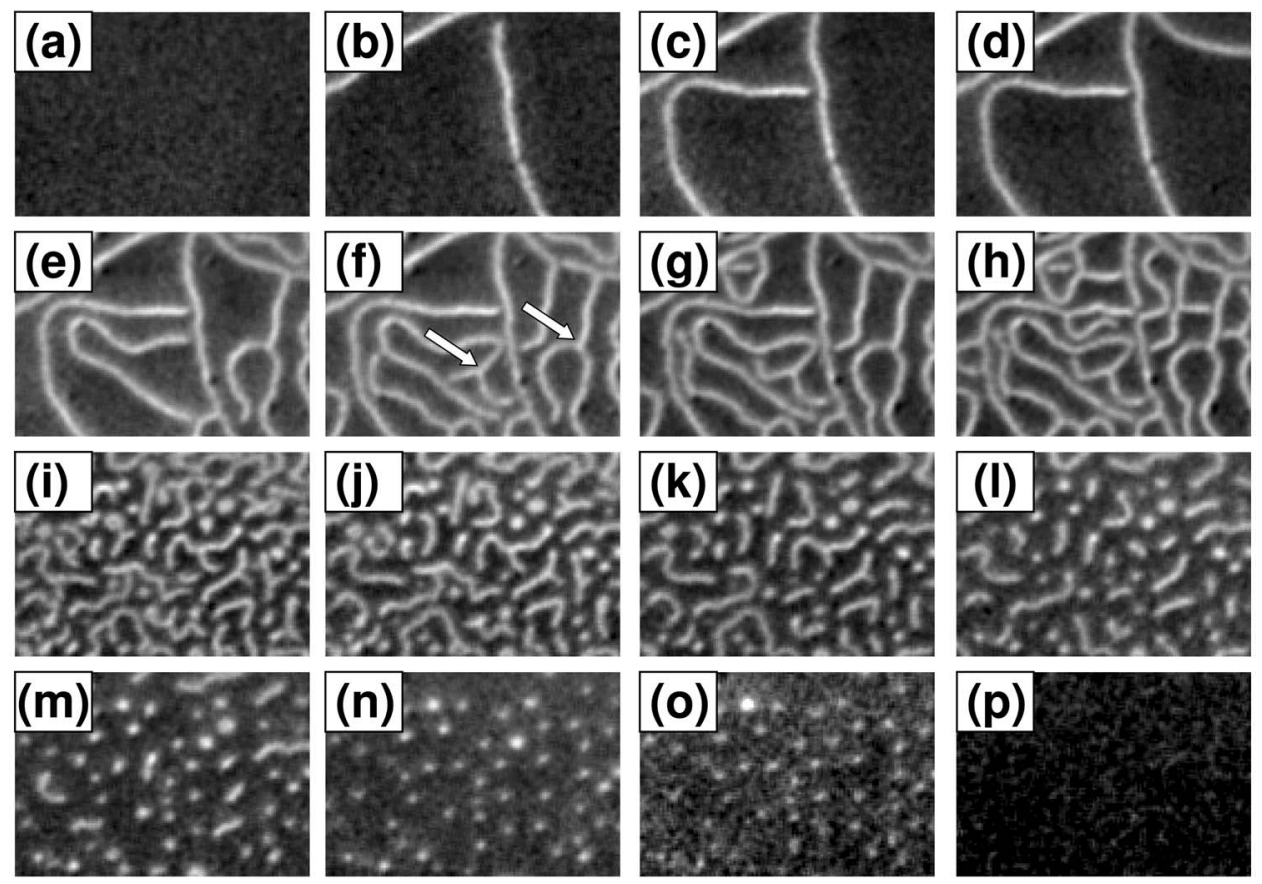
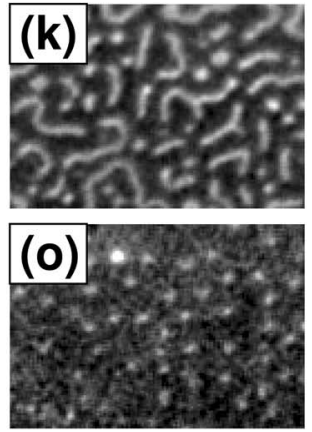
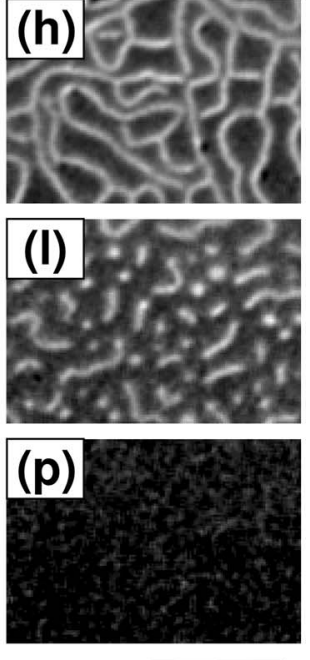

FIG. 1. (a)-(h) Patterns obtained at $T=5 \mathrm{~K}$ with decreasing field from $\mu_{0} H_{z}=38.4 \mathrm{mT}$ to $\mu_{0} H_{z}=35.6 \mathrm{mT}$ in steps of $0.4 \mathrm{mT}$ and (i)-(p) with increasing field from $\mu_{0} H_{z}=35.9 \mathrm{mT}$ to $\mu_{0} H_{z}$ $=38.7 \mathrm{mT}$ in steps of $0.4 \mathrm{mT}$. White (black) indicates SC (normal) regions. bubble structure, at low fields, is associated with the presence of a diamagnetic band formed at the edge of the sample that prevents continuous penetration of flux. At higher fields, but still well below $H_{c}$, the diamagnetic band disappears, the bubble pattern becomes less favorable and a stripe pattern is formed. Another characteristic of flux bubble patterns is that the surface tension, associated with the positive interface energy between $\mathrm{N}$ and $\mathrm{SC}$ domains, prevents the formation of very small bubbles. Magneto-optic experiments ${ }^{13}$ on $\mathrm{Pb}$ and In show that the diameter of the flux bubbles is practically field independent. This means that the size of the bubbles is determined by the competition between the interaction of the current flowing around the bubble and the interface energy and is independent of the magnetic interaction between domains.

In the opposite limit, that is close to the transition to the normal state, a SC laminar pattern is observed upon flux exit when decreasing the field from above $H_{c}$. In samples of thickness between 1 and $10 \mu \mathrm{m}$ (Refs. 14 and 17) threads of SC stripes are formed abruptly when the field is decreased while small SC domains (bubbles) are formed when increasing the field. In Ref. 17 it is argued that the IS stripe pattern formed when decreasing the field is a consequence of an elliptical instability of bubble domains induced by long range dipolar interactions.

Thus, it has been shown that the overall topological characteristics of the observed flux patterns can be well described using the present theoretical models. However, there are still open questions as for example (i) what is the equilibrium topology close to $H_{c}$ (stripe or bubble), (ii) how is the local evolution with field or temperature of the patterns (either stripes or bubbles) formed close to $H_{c}$, and (iii) how reproducible are the observed structures. In this work we present results on the patterns formed at fields near $H_{c}$ (low SC fraction). We follow in detail the evolution of the structures as the field is changed and test their stability by applying a temporal oscillating magnetic field. Once a stable configuration is obtained close to $H_{c}$, we study the influence of a changing external field on the topology of the IS. The effects of pinning on the observed structures are discussed.

The sample for which we present results here, was obtained by flattening a $\mathrm{Pb}$ foil down to a thickness of $0.15 \mathrm{~mm}$ and cutting it in a circular shape with a diameter of $5 \mathrm{~mm}$. After cutting to the desired dimensions, the sample was annealed at $250{ }^{\circ} \mathrm{C}$ in order to remove defects possibly induced during flattening and cutting. The critical temperature of this sample is $T_{c}=7.2 \mathrm{~K}$. In all the experiments presented here the magnetic field is applied in the $z$ direction (perpendicular to the largest surface of the sample). By means of magnetooptical imaging, ${ }^{18}$ maps of the local magnetic field, $B_{z}$, just above the surface of the sample are obtained. All the experiments were made at constant temperature in the range $3.5 \leqslant T \leqslant 6.5 \mathrm{~K}$. We show only results at $T=5 \mathrm{~K}$ since we observed similar behavior in the whole range of studied temperatures. Throughout this paper the superconducting domains are shown in the images as bright regions while the normal ones are shown dark.

\section{RESULTS AND DISCUSSION}

In Fig. 1 we show the evolution of the domain structure at $T=5 \mathrm{~K}$ as a function of decreasing magnetic field [(a)-(h)] and increasing field $[(\mathrm{i})-(\mathrm{p})]$ after zero field cooling the sample. ${ }^{19}$ The images correspond to fields close to the transition field $H_{c}$. At high enough fields, the sample is normal everywhere, see Fig. 1(a). As the field is decreased, superconductivity nucleates in the form of stripes [Fig. 1(b)] that grow abruptly in apparently random directions. ${ }^{14,17}$ This 


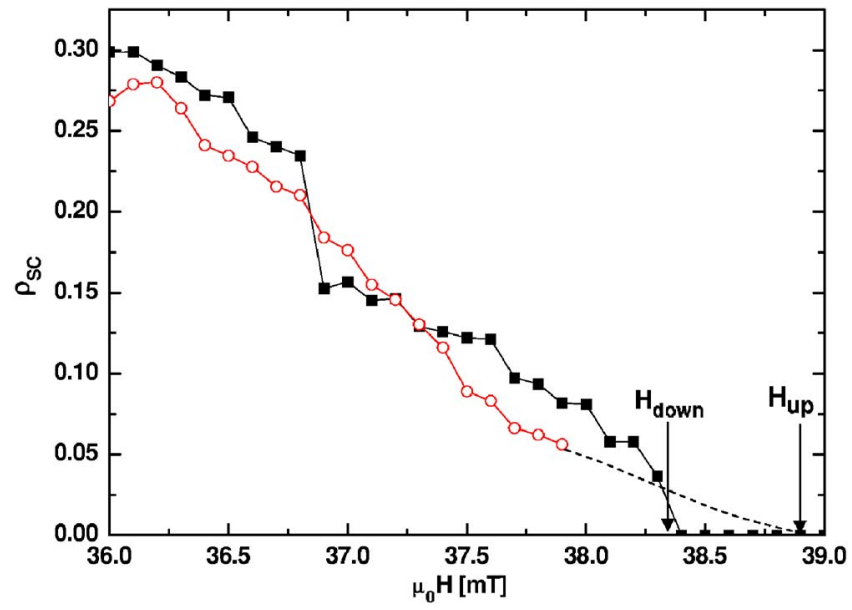

FIG. 2. (Color online) Fraction of SC domains as a function of field at $T=5 \mathrm{~K}$. The filled squares (open circles) correspond to a decreasing (increasing) field experiment. The values of $H_{\text {down }}$ and $H_{\text {up }}$ for this experiment are indicated. In the increasing field experiment the contrast of the images decreases as $H \rightarrow H_{\text {up. Thus, it is not }}$ possible to determine unequivocally the values of $\rho_{\mathrm{SC}}$ in that limit. In this case, the lowest value well defined is 0.05 . The dashed line is an extrapolation of $\rho_{\mathrm{SC}}$ up to $H_{\mathrm{up}}$.

leads to jumps in the fraction of SC domains, $\rho_{\mathrm{SC}}$, as a function of decreasing field (see Fig. 2, filled squares). At the jumps, new stripes are nucleated and sometimes branching of stripes is observed. This process finally gives rise to a globally disordered and labyrinthine structure [Fig. 1(h)]. The pattern is a result of the frustration of the system in trying to fill the space with stripes that repel each other, in combination with pinning of already formed SC domains. If pinning were weak then one could expect that the fraction of SC domains would grow by curling, bending or even widening of the already nucleated stripes. However, that is not observed and instead the already nucleated stripes remain mainly static as the field changes. The growth mechanism of the SC phase is different from the growth process of the $\mathrm{N}$ phase in the low field regime. In that case, magneto-optical images show ${ }^{13}$ movement of flux bubbles at very low fields and both jumping and widening of flux stripes as the density of normal domains increases with increasing field.

It is interesting to note that the branching of stripes occurs in such a way that only three fold vertices are formed [see arrows in Fig. 1(f)]. Similar behavior is observed in ferrimagnetic garnet films. ${ }^{20}$ Besides branching of domains, breaking of some stripes is also observed, see Figs. 3(a) and 3(b). In this case the fission of the SC stripe B is caused by
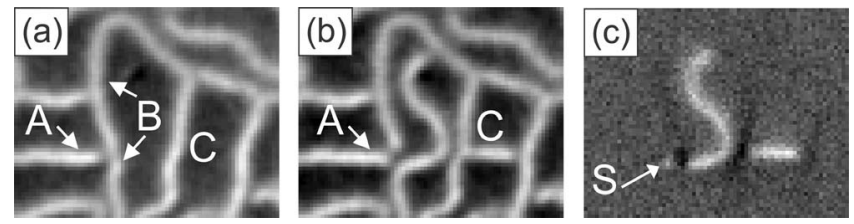

FIG. 3. Sequence for decreasing field from $36 \mathrm{mT}$ (a) to $35.6 \mathrm{mT}$ (b) at $T=5 \mathrm{~K}$ that shows breaking of one stripe (B). (c) Subtraction of images (b) and (a). the elongation of the almost horizontal segment $\mathrm{A}$ that pushes (due to the repulsive interaction) the stripe $\mathrm{B}$, inducing the cutting and subsequently propagation of the SC domain in the form of a s-shaped stripe. Moreover, in a similar way this new s-shaped segment pushes to the right the stripe $\mathrm{C}$ provoking branching of that stripe. This sequence of events is more evident in Fig. 3(c) that shows the substraction of the images shown in (a) and (b). The small change in the length of segment $A$, that causes the change in the structure, is evident as a small bright spot (S) next to the lower left end of the new s-shaped domain.

The transition from the IS to the NS with increasing $H$ is shown in the sequence Figs. 1(i)-1(p). Initially, the pattern consists of short and branched SC stripes and some SC bubbles. As the field is increased some branches disappear and in other cases the fission of one single stripe into bubbles is observed. Finally, only bubbles are left, that start to fade away as the transition to the NS is approached. The behavior of $\rho_{\mathrm{SC}}$ as a function of field in this experiment is shown in Fig. 2 (open circles). As the system approaches the NS the contrast in the images decreases. Thus, even though it is possible to determine at which field the sample is in the normal state, it is very difficult to obtain reliable values of $\rho_{\mathrm{SC}}$ when $\rho_{\mathrm{SC}} \rightarrow 0$.

From our experiments, and in agreement with previous reported results, ${ }^{14,17}$ we find that the magnitude of the field at which the first SC stripe nucleates with decreasing field differs from the value at which the last SC domain is still detected with increasing field. We define the corresponding transition fields as $H_{\text {down }}$ and $H_{\text {up }}$, see Fig. 2. The transition always occurs at a larger value with increasing field than in the opposite direction. Average values for our samples are $\mu_{0} H_{\text {up }}=38.6 \pm 0.4 \mathrm{mT}$ and $\mu_{0} H_{\text {down }}=37.8 \pm 0.6 \mathrm{mT}$ at $T=5 \mathrm{~K}$. Thus, the width of the irreversibility region, attributed to a supercooling of the normal phase, ${ }^{17}$ is $\mu_{0} \Delta H$ $=\mu_{0} H_{\text {up }}-\mu_{0} H_{\text {down }} \sim 1 \mathrm{mT}$. This difference seems to slightly decrease when the temperature increases. In our case the irreversibility region is approximately $2.5 \%$ of the value of $H_{c}$ while in the case of thin In samples ${ }^{17}$ it is of the order of $15 \%$.

Besides the irreversibility in fields, it is observed that the transition NS $\rightarrow$ IS is abrupt since SC regions nucleate in an avalanchelike manner, while the transition in the opposite direction is smooth, ${ }^{10,14,17}$ see Fig. 2. The nucleation of SC regions is abrupt even when the field is swept at the smallest rate available in our experiment, $\mu_{0} d H / d t=1 \mathrm{mT} / \mathrm{min}$. The abrupt nucleation of SC domains is similar to what is observed in the formation of magnetic domains in multilayers of $\mathrm{Co} / \mathrm{Pt}^{21}$ In that case, a jump in the magnetization curve has been associated with the avalanchelike propagation of stripe domains. However, when the field is increased towards saturation it is observed that the magnetization changes smoothly. Numerical simulations ${ }^{22}$ in this system show that the magnetization jumps, and the consequent avalanche process, only occur if the disorder in the system was lower than some critical amount. If the disorder is too large, the domains are pinned more easily preventing the propagation of domains. Analogously, we conjecture that the avalanche of SC domains in a normal matrix can also be influenced by the presence of defects that pin the N-SC interfaces. 
From the sequence of images of the formation of the IS (Fig. 1) we observe that the stripe width is roughly constant as the field is changed. When increasing the field towards $H_{\text {up }}$ the width of the SC stripes is $(39 \pm 9) \mu \mathrm{m}$ (the error comes from the resolution of our images) while in the decreasing field experiment the width is $(42 \pm 9) \mu \mathrm{m}$. As a first approximation the width of the SC domains can be calculated in the frame of the Landau theory for the laminar structure. ${ }^{9,23}$ In this simple model the terms considered in the calculation of the free energy are, the wall energy associated with the interfaces, the energy of the nonuniform magnetic field outside the SC and an excess in energy due to the broadening of the normal domains at the surface of the sample. From the minimization of energy the period of the domain structure is

$$
a=[\delta(T) \cdot d / f(h)]^{1 / 2},
$$

where $\delta(T)$ is the interface width or wall energy parameter, $d$ is the thickness of the sample, $h$ is the reduced field defined as $H / H_{c}$ and $f(h)$ is a function that has a peak at $h \sim 0.5$ and goes smoothly to 0 for $h \rightarrow 0$ and $h \rightarrow 1$. The limiting form of this function close to $h=1$ is ${ }^{23}$

$$
f(h)=\frac{\ln 2}{\pi}(1-h)^{2},
$$

$a$ can be expressed as a function of the width of $\mathrm{N}$ and SC domains as $a=a_{\mathrm{N}}+a_{\mathrm{SC}}$ and $a_{\mathrm{N}} / a=H / H_{c}=h$. Thus, the width of the SC domains can be written as $a_{\mathrm{SC}}=(1-h) \cdot a$. Using Eqs. (1) and (2) one finds that the width of SC regions close to $h=1$, within the Landau model, is independent of $h$ (consistent without experiment) and given by

$$
a_{\mathrm{SC}}^{L}=\sqrt{\frac{\delta d \pi}{\ln 2}} .
$$

Considering the empirical law $\delta(T)=\delta(0) / \sqrt{\left[1-\left(T / T_{c}\right)\right]}$ with $\delta(0)=0.056 \mu \mathrm{m}$ for $\mathrm{Pb}$ (Ref. 9) and $d=150 \mu \mathrm{m}$ we expect (for $T=5 \mathrm{~K}$ ) $a_{\mathrm{SC}}^{L}=8.3 \mu \mathrm{m}$, roughly 5 times smaller than the observed value, $a_{\mathrm{SC}}^{\exp } \sim 40 \mu \mathrm{m}$ (either in decreasing or increasing field experiments).

Close to $H_{c}$ the $\mathrm{CL}$ model predicts $^{12} a_{\mathrm{SC}}^{\mathrm{CL}}$ $\simeq\{2 \pi \delta(T) \cdot d / \ln [0.71 /(1-h)]\}^{1 / 2}$. From our experiments we can determine the field at which SC nucleates and using tabulated value for the critical field of $\mathrm{Pb}, \mu_{0} H_{c}(T=0)$ $=80.3 \mathrm{mT},{ }^{24}$ we find $h_{\text {nucl }}=h_{\text {down }}=0.91$. Then, at the nucleation field we find that $a_{\mathrm{SC}}^{\mathrm{CL}} \simeq 6.8 \mu \mathrm{m}$ is rather close to the Landau model result and approximately 6 times smaller than our experimental result. In addition, this model predicts that $a_{\mathrm{SC}}^{\mathrm{CL}} \rightarrow 0$ for $h \rightarrow 1$ while experimentally we do not observe a field dependence of the stripe width. At this point, it is important to mention that although the thickness of the sample used in our experiments is above the theoretically calculated value $^{9}$ for the beginning of the branching regime, the observed patterns show no evidence of branching. This branching regime should not be confused with the branching of SC stripes as we mentioned when describing the patterns shown in Fig. 1. Here, we refer to the branching of the normal regions ${ }^{9}$ as a consequence of a widening of the domains as they approach the surface of the sample. Both the Landau model and the CL model do not take into account branching. Thus, the discrepancy between the experimental values of the SC stripe width and the theoretical predictions might be due to the fact that our sample is close to the branching transition.

Within the framework of the CCL model it is proposed ${ }^{17}$ that the stripe pattern observed near $H_{c}$ is a result of an elliptical instability of circular domains. This gives a possible way to estimate the width of the stripes when the IS is formed. Assume that a single cylindrical SC domain is formed when decreasing field. If the diameter of the cylinder is larger than the critical diameter for the bubble elliptical instability to occur, then the bubble will evolve as an elongated domain (stripe). In that case, the observed width of the stripe should be similar to the diameter of the bubble nucleated in the onset of domain formation. Within the CCL model the free energy of an isolated SC cylindrical domain of radius $R$ can be written as ${ }^{17}$

$$
F=2 \pi \sigma_{\mathrm{SN}} d^{2}\left(\frac{p}{2}+B_{m}\left(h^{2}-1\right) \pi \frac{p^{2}}{4}+B_{m} h^{2} \frac{p^{3}}{3}\right),
$$

where $p=2 R / d$ is the reduced diameter of the tube, $\sigma_{\mathrm{SN}}$ is the surface tension of the N-SC interface and $B_{m}=d / 2 \pi \delta$ is the magnetic Bond number that corresponds to the ratio between magnetic and surface forces. The first and second terms are the same energy contributions as considered by Landau. $^{7}$ The correction introduced by the CCL model ${ }^{13}$ is the third term, which represents the interaction energy of the screening current flowing in the surface of the bubble. It is important to mention that the free energy written above is a valid expression only near the N-IS transition since it corresponds to the energy of an isolated SC domain.

Minimization of this energy gives the equilibrium reduced diameter of a SC bubble formed at the nucleation field $h_{\text {nucl }}$ as $p_{0}=y\left[1+(1-8 / z)^{1 / 2}\right]$ where $y=\pi\left(1-h_{\text {nucl }}^{2}\right) /\left(4 h_{\text {nucl }}^{2}\right)$ and $z=\pi^{2}\left(1-h_{\text {nucl }}^{2}\right)^{2} B_{m} / h_{\text {nucl }}^{2}$. Inserting $h_{\text {nucl }}=h_{\text {down }}=0.91$ and $B_{m}=d /(2 \pi \delta)=233$ for our sample, we obtain $p_{0}=0.32$ that corresponds to a bubble diameter of $48 \mu \mathrm{m}$. From linear instability analysis ${ }^{25}$ the critical diameter for the elliptical instability is $3 d / h \sqrt{2 B_{m}}$. In the present case this value corresponds to a diameter of $22.9 \mu \mathrm{m}$. Thus, it is expected that at $h_{\text {down }}$ the instability will develop and a stripe with a width of the order of the equilibrium bubble diameter $(48 \mu \mathrm{m})$ will be formed. This result is in good agreement with the (42 \pm 9$) \mu \mathrm{m}$ width of SC stripes observed in the present experiment when decreasing the field. This similarity suggests that when the elliptical instability develops the SC domain preserves the equilibrium diameter of a bubble at the nucleation field in one direction and elongates in the perpendicular one. The fact that the SC stripe width is the same in decreasing and increasing field experiments supports the idea that, despite the presence of pinning, the observed width corresponds to an equilibrium situation. However, the observed topology (stripes) when decreasing the field is a consequence of the nucleation and growth process of SC domains in a normal matrix.

To gain more insight in the effects of pinning when the IS is formed, we study the reproducibility of the observed pat- 

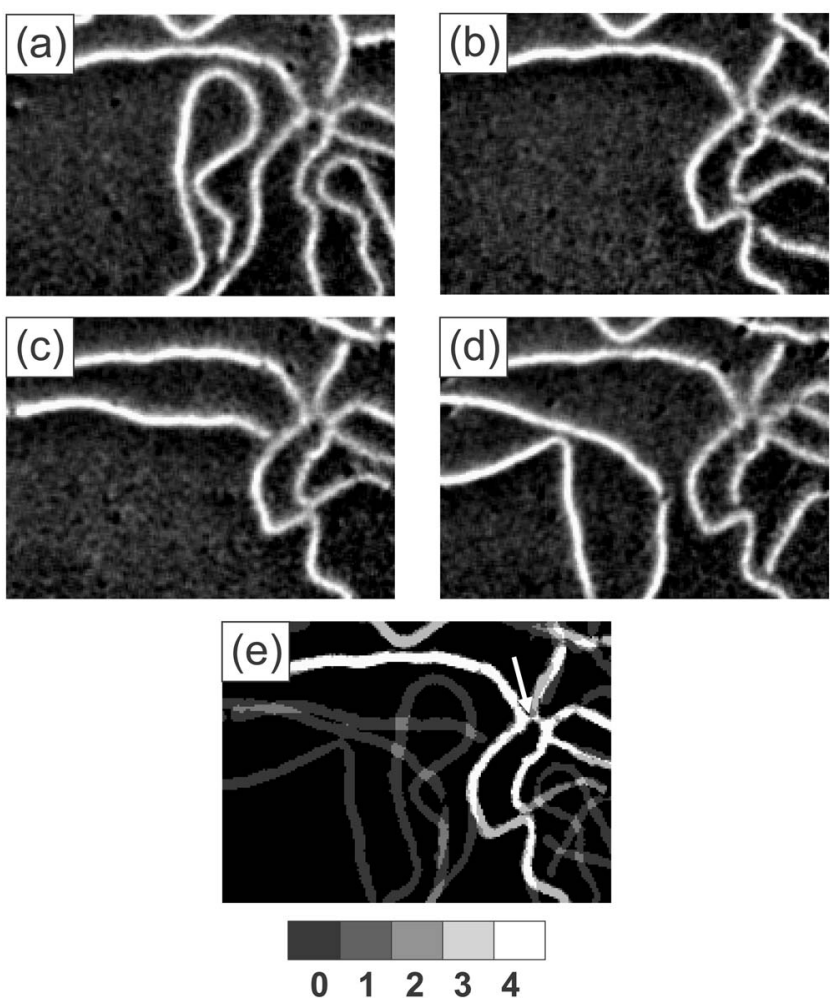

FIG. 4. (a)-(d) Patterns formed in the same region of the sample in four different experiments starting from above $\mu_{0} H_{\text {up }}$ at $\mu_{0} H=37 \mathrm{mT}$ and $T=5 \mathrm{~K}$. (e) Superposition of binary images corresponding to the four images shown above, brighter regions indicate more reproducibility.

terns. We repeat many times a decreasing field experiment and observe the structures nucleated in each experiment. The results of four different realizations in a certain region of the sample are shown in Figs. 4(a)-4(d). In each case the image was taken at $\mu_{0} H=37 \mathrm{mT}, T=5 \mathrm{~K}$ and the field was always decreased at $100 \mathrm{mT} / \mathrm{min}$, from far above $H_{\text {up }}$. The sequence of images as the field is continuously decreased (not shown) reveals that, in each realization, the SC phase nucleates al- ways in the same spot in the sample [see arrow in Fig. 4(e)]. However, the further growth and branching of domains lead to different final states. Evidently, the SC fraction is not the same every time suggesting that these structures are out of equilibrium. In Fig. 4(e) the patterns corresponding to the four different experiments shown in (a)-(d) are superimposed. The arrow points to the spot where the SC domains start to grow in the different experiments and the gray scale indicates the degree of superposition where white corresponds to the maximum (that is, where a SC domain is present in all four experiments). The reproducibility of the location of the first nucleated stripe in Fig. 4 makes evident that there can be preferred locations where superconductivity is locally enhanced. In general, the subsequent stripes appear in different locations when repeating the experiment. This indicates that the distribution of pinning sites allows the formation of locally different structures. The fact that small perturbations in the initial state lead to different structures points to an intrinsic instability of the system when the stripe pattern is growing. This result is consistent with the proposed $^{17}$ elliptical instability as an explanation for the topological hysteresis.

As we mentioned above we have some evidence that the stripe pattern formed close to the $H_{c}$ line does not correspond to an equilibrium configuration. In order to test the stability of these patterns we follow their evolution in the presence of a magnetic field that is periodically modulated in time. The experimental sequence is the following. First, a large magnetic field, $H_{z}>H_{\text {up }}$ is applied at low temperature, $T<T_{c}$. Then, the field is decreased until some stripes are nucleated. After that, the modulation of field starts. In Fig. 5 we show the evolution of the structure at the highest magnetic field of the oscillation as a function of time. In this case, the triangular oscillation was done between $\mu_{0} H_{\min }=35.5 \mathrm{mT}$ and $\mu_{0} H_{\max }=37.5 \mathrm{mT}$ with a frequency of $0.21 \mathrm{~Hz}$ and at $5 \mathrm{~K}$.

The first time the magnetic field is decreased down to $H_{\text {max }}$ the sample is in the normal phase in the region of sample shown in the figure. The first image in Fig. 5 is therefore shown after the first cycle of the field and the next images are taken every 15 cycles of the field. The topology
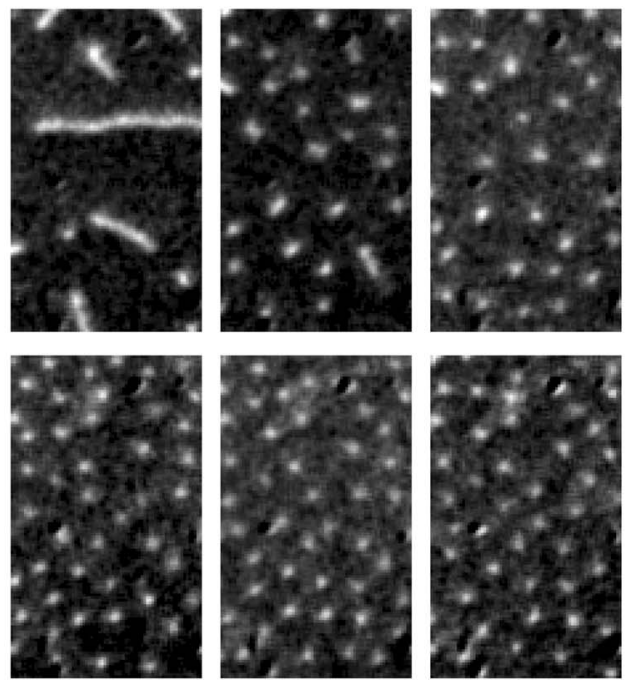
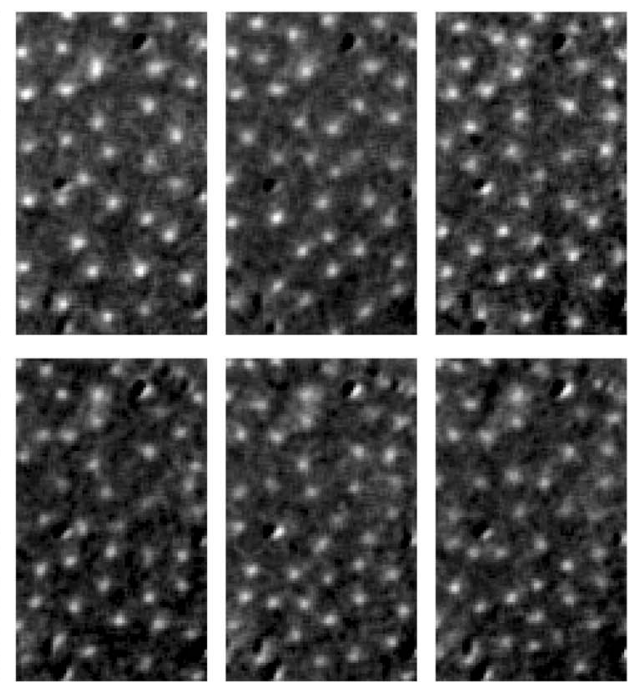

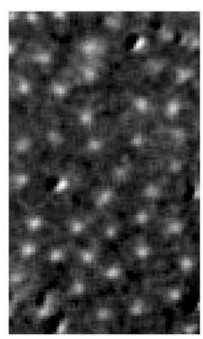

FIG. 5. Temporal evolution of the spatial distribution of SC domains (white) as a function of magnetic field cycling at $T=5 \mathrm{~K}$. The modulation is made at $0.21 \mathrm{~Hz}$ and between 36.5 and $37.5 \mathrm{mT}$. The images are taken every 15 cycles of the modulation at $\mu_{0} H_{\max }=37.5 \mathrm{mT}$. 


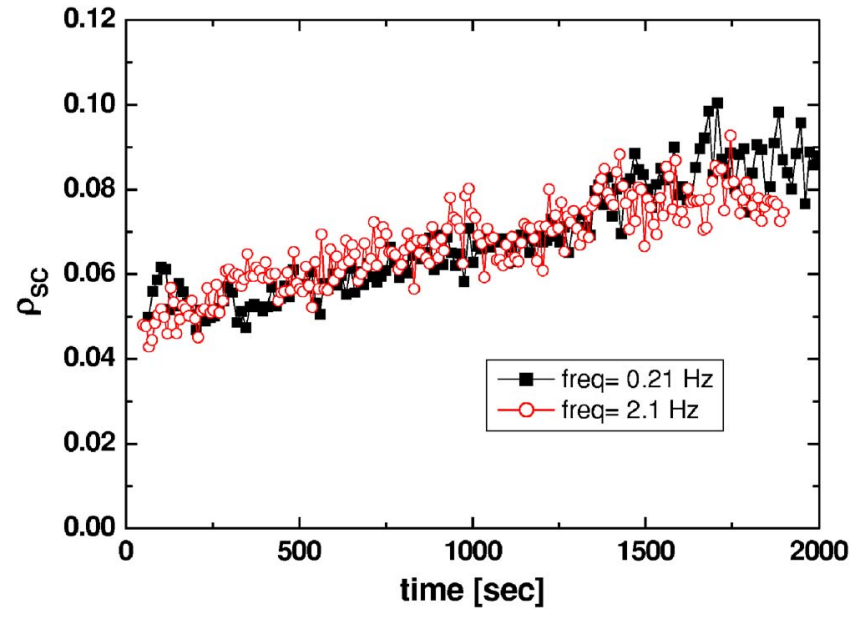

FIG. 6. (Color online) Fraction of SC domains, $\rho_{\mathrm{SC}}$, as a function of time for two different frequencies of oscillation.

of the initial pattern depends on the value of the field, $H_{\max }$, and on the specific configuration of stripes formed while decreasing the magnetic field. In the experiment we present here, the structure after the first cycle of the modulation consists of short segments coexisting with some bubbles. We observe that a pattern of only bubbles is already formed after 30 cycles of the field. The fraction of SC domains, $\rho_{\mathrm{SC}}$, increases as a function of time as shown in Fig. 6. The behavior is the same for two different frequencies of oscillation $(0.21$ and $2.1 \mathrm{~Hz})$. From our experiments we can conclude that the structure after many field cycles depends on $H_{\max }$ and not very much on the amplitude or frequency of the modulation, at least for the low modulation frequencies $(0.1 \mathrm{~Hz}-2.1 \mathrm{~Hz})$ studied in the present case. The diameter of bubbles after the modulation is $D=42.2 \pm 8.0 \mu \mathrm{m}$ (here the error correspond to the standard deviation of the distribution of diameters). This value can be compared with the diameter of bubbles observed in the increasing field experiment as described at the beginning of the section, see, for example, Fig. 1(n). In that case we find that $D=45.6 \pm 16.7 \mu \mathrm{m}$. Although the average values are similar in both cases, it is important to note that the distribution of sizes is much broader in the case of increasing the field towards $H_{\text {up }}$ than after the modulation experiment. The fact that a bubble pattern is stabilized after cycling the field and that the density of bubbles increases with time indicate that the pattern composed of SC bubbles is closer to equilibrium than the long SC stripes obtained just decreasing the field. This is further confirmed by performing small modulations of the magnetic field of amplitude $\Delta H$ of the order of $1 \%$ of the field at which the bubble pattern is observed, $H_{\max }$. In this experiment only small displacements of the bubbles but no change in topology are observed. It is reasonable to think that pinning contributes to stabilize this structure and prevents the formation of a truly equilibrium structure (a hexagonal lattice).

It is interesting to see how the bubble pattern evolves as the field is decreased, see Fig. 7. It is evident from the sequence of images that the superconducting bubbles elongate as the field is decreased leading to the formation of short
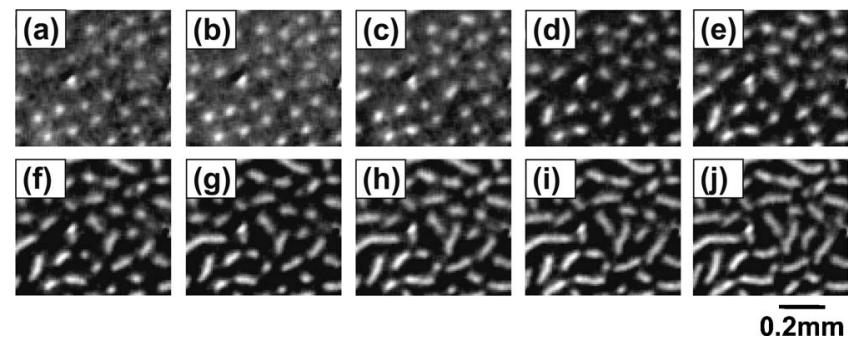

FIG. 7. Evolution of the bubble pattern generated by modulation of field, with decreasing magnetic field at $T=5 \mathrm{~K}$. The images correspond to (a) $\mu_{0} H_{z}=37.4 \mathrm{mT}$ to (j) $\mu_{0} H_{z}=35.6 \mathrm{mT}$ in steps of $0.2 \mathrm{mT}$.

stripes oriented in different directions. Since not all the bubbles elongate in the same manner for a small step of field, coexistence of stripes and bubbles is observed [Figs. 7(e) and 7(f)]. As the field is further decreased the segments start to bend and curl as a result of the repulsive interaction between them. We can identify the beginning of this topological transition at $\mu_{0} H=37 \mathrm{mT}$ where the bubbles start to elongate. At $\mu_{0} H=36 \mathrm{mT}$ a great majority of domains are stripes. Close to the transition field the bubble diameter is $47.8 \pm 9.2 \mu \mathrm{m}$.

In Fig. 8 we show the fraction of SC domains, $\rho_{\mathrm{SC}}$, as a function of field in two different situations, one for decreasing field from the normal state (filled squares) and the other decreasing field after the modulation of the field (open circles). The first case corresponds to an experiment as the one shown in Figs. 1(a)-1(h). In that case, as discussed above, $\rho_{\mathrm{SC}}$ changes abruptly as the field is decreased. The modulation experiment is the same as in the case of Fig. 5 ( $H$ was oscillated between $\mu_{0} H_{\min }=35.5 \mathrm{mT}$ and $\mu_{0} H_{\max }$ $=37.5 \mathrm{mT}$ with a frequency of $0.21 \mathrm{~Hz}$ and $T=5 \mathrm{~K}$ ). The two snapshots in Fig. 8 show patterns in these two situations at the same applied field. It is interesting to see that even though the values of $\rho_{\mathrm{SC}}$ are very similar in both cases the topologies are clearly different. After the modulation there are many small domains, thus the surface energy term is

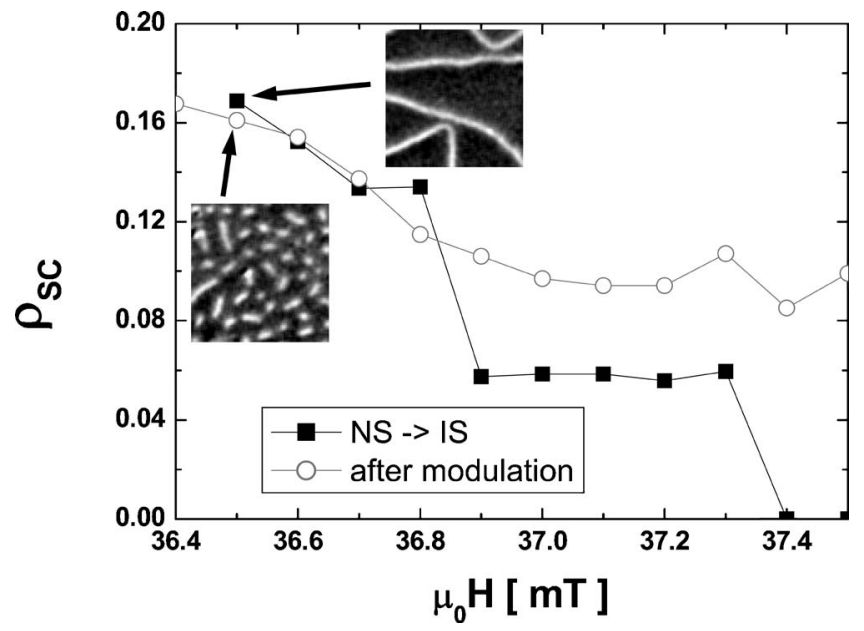

FIG. 8. Fraction of SC domains as a function of field at $T=5 \mathrm{~K}$ following two different paths: decreasing field crossing the $\mathrm{N}$-IS line and decreasing field starting from the bubble phase after modulation of magnetic field. 
larger when compared to the structure of large stripes. On the other hand, the distribution of magnetic field inside the sample is more homogeneous diminishing the magnetic energy term.

The size of flux bubbles (N-bubbles in a SC matrix) has been associated ${ }^{6}$ with the competition between surface tension and the self-interaction of the screening current. A similar argument should apply to the present case of SC bubbles since both energy terms are related with the interface N-SC, and in that sense the problem is symmetric. However, in the case of flux bubbles the flux conservation constraint impedes the continuous and reversible growth of bubbles. Since there is no such condition in the case of SC bubbles, they can grow continuously and reversible as observed in the experiments. Thus, the conservation of flux imposes an intrinsic asymmetry when considering flux and superconducting bubbles. ${ }^{26}$ We observe that once we drive the system close to equilibrium, the growth mechanism of the SC phase is rather different than when nucleating the metastable IS from the normal state. Now the increase of SC regions is achieved by elongation of domains instead of nucleating new ones.

\section{CONCLUSIONS}

Our magneto-optical experiments show that with decreasing field from far above $H_{c}$, the formation of the IS takes place in the form of avalanches of SC stripes. The stripes grow in random directions and remain mainly pinned as the field is changed. The growth of the superconducting fraction in the IS takes place mainly by the appearance of new stripes and by branching of already present stripes. A labyrinth pattern is formed as the new stripes curl more since the space free of stripes becomes smaller as the field is decreased. We have shown that the labyrinthine pattern is not locally fully reproducible indicating that the pinning of the stripes is not due to the presence of extended defects. When the field is increased towards $H_{c}$ short SC stripes and bubbles coexist.
Finally, a bubble pattern is left that smoothly disappears as the sample becomes normal.

It is interesting to point out that even though the Landau model was developed for equilibrium configurations well below the branching transition we observe a field independent SC stripe width consistent with the prediction of this model and at variance with the more sophisticated CL and CCL models. Minimizing the free energy of a isolated SC circular domain within the CCL model gives a value for the equilibrium bubble diameter that is very similar to our experimentally observed SC stripe width. We also find that the SC stripe width is the same for increasing and decreasing field in the region close to $H_{c}$ suggesting that the observed width correspond to the equilibrium value. Modulation in time of the magnitude of the magnetic field shows that a pattern formed by SC bubbles is closer to equilibrium than the stripe pattern. The bubble diameter and stripe width are the same and independent of the modulation history, reinforcing the idea that this is an equilibrium size.

Moreover, we have been able to observe the growth mechanism of SC stripes starting from bubble phase. The bubbles elongate, bend and sometimes branch as the field is decreased. Coexistence of bubbles and short stripes is observed. A similar transition but of normal domains has been proposed and observed experimentally. ${ }^{6,13}$ However, no prediction has been made for the case of SC domains. Thus, the experimental observations presented here provide a new testing ground for theoretical models, in particular the CCL model.

\section{ACKNOWLEDGMENTS}

The authors would like to thank T. Arion for his contribution in the early stage of the experiments present here. This work was supported by FOM (Stichting voor Fundamenteel Onderzoek der Materie) which is financially supported by NWO (Nederlandse Organisatie voor Wetenschappenlijk Onderzoek).

\footnotetext{
*Present address: INPAC-Institute for Nanoscale Physics and Chemistry, Nanoscale Superconductivity and Magnetism and Pulsed Fields Group, Katholieke Universiteit Leuven, Celestijnenlaan 200D, Leuven B-3001, Belgium.

${ }^{1}$ M. Seul and D. Andelman, Science 267, 476 (1995) and references therein.

${ }^{2}$ T. Garel and S. Doniach, Phys. Rev. B 26, 325 (1982).

${ }^{3}$ T. C. Halsey, Phys. Rev. E 48, R673 (1993).

${ }^{4}$ M. Seul, Europhys. Lett. 28, 557 (1994).

${ }^{5}$ F. Elias, C. Flament, J.-C. Bacri, and S. Neveu, J. Phys. I 7, 711 (1997).

${ }^{6}$ V. Jeudy, C. Gourdon, G. Karczewski, and T. Le Anh, Physica C 388, 775 (2003).

${ }^{7}$ L. D. Landau, Zh. Eksp. Teor. Fiz. 7, 371 (1937).

${ }^{8}$ Y. V. Sharvin, Sov. Phys. JETP 6, 1031 (1958).

${ }^{9}$ R. P. Huebener, Magnetic Flux Structures of Superconductors (Springer-Verlag, New York, 1990).
}

${ }^{10}$ D. E. Farrell, R. P. Huebener, and R. T. Kampwirth, J. Low Temp. Phys. 19, 99 (1975).

${ }^{11}$ R. N. Goren and M. Tinkham, J. Low Temp. Phys. 5, 465 (1971).

${ }^{12}$ R. E. Goldstein, D. P. Jackson, and A. T. Dorsey, Phys. Rev. Lett. 76, 3818 (1996); A. T. Dorsey and R. E. Goldstein, Phys. Rev. B 57, 3058 (1998).

${ }^{13}$ A. Cēbers, C. Gourdon, V. Jeudy, and T. Okada, Phys. Rev. B 72, 014513 (2005).

${ }^{14}$ R. P. Huebener, R. T. Kampwirth, and V. A. Rowe, Cryogenics 12, 100 (1972).

${ }^{15}$ V. Jeudy, C. Gourdon, and T. Okada, Phys. Rev. Lett. 92, 147001 (2004).

${ }^{16}$ R. Prozorov, R. W. Giannetta, A. A. Polyanskii, and G. K. Perkins, Phys. Rev. B 72, 212508 (2005).

${ }^{17}$ C. Gourdon, V. Jeudy, and A. Cebers, Phys. Rev. Lett. 96, 087002 (2006).

${ }^{18}$ R. J. Wijngaarden, K. Heeck, M. Welling, R. Limburg, M. Pan- 
netier, K. van Zetten, V. L. Roorda, and A. R. Voorwinden, Rev. Sci. Instrum. 72, 2661 (2001).

${ }^{19}$ Similar structures are observed if after decreasing the field to an arbitrary value (even down to 0 ) the field is increased without zero field cooling the sample.

${ }^{20}$ G. Portman, A. Vateriaus, and D. Pescia, Nature (London) 422, 701 (2003).

${ }^{21}$ J. E. Davies, O. Hellwig, E. E. Fullerton, G. Denbeaux, J. B. Kortright, and K. Liu, Phys. Rev. B 70, 224434 (2004).
${ }^{22}$ M. S. Pierce et al., Phys. Rev. Lett. 94, 017202 (2005); E. A. Jagla, Phys. Rev. B 72, 094406 (2005).

${ }^{23}$ L. D. Landau and E. M. Lifshitz, Electrodynamics of Continuous Media (Pergamon, Oxford, 1960).

${ }^{24}$ P. G. de Gennes, "Superconductivity of metals and alloys," Frontiers in Physics (W.A. Benjamin, New York, 1966).

${ }^{25}$ A. Cebers and P. A. Janmey, J. Phys. Chem. 106, 12351 (2002).

${ }^{26}$ R. Choksi, R. V. Kohn, and F. Otto, J. Nonlinear Sci. 14, 119 (2004). 